\title{
Facilitated pointing to remembered objects in front: Evidence for egocentric retrieval or for spatial priming?
}

\author{
Jonathan W. Kelly and Timothy P. MCNAMaRa \\ Vanderbilt University, Nashville, Tennessee
}

\begin{abstract}
Recent spatial memory theories propose that long-term spatial memories are retrieved egocentrically. One source of evidence comes from imagined perspective taking, in which participants learn an object layout, later imagine standing at one object and facing a second (orienting) object, and then point to a third (target) object from the imagined perspective. Pointing is faster for target objects in the anterior than in the posterior half of imaginal space. This "front facilitation" is consistent with asymmetric sensory and biomechanical body properties (favoring the anterior half of body space), supporting claims of egocentric retrieval. However, front facilitation might actually result from spatial priming: Proximity differences might cause orienting objects to prime target objects more in the anterior than in the posterior half of imagined space. Using a modified perspectivetaking task that unconfounded front facilitation and spatial priming, two experiments identified separate influences of front facilitation and spatial priming when participants imagined perspectives within the surrounding environment or a remote environment.
\end{abstract}

Spatial behaviors, such as finding one's car in a parking lot, depend on long-term spatial memories, and the organizational properties of those memories have received considerable research attention. The preponderance of evidence indicates that spatial memories are orientation dependent, organized around a small number of reference directions centered on the environment (Kelly \& McNamara, 2008b; Mou \& McNamara, 2002; Shelton \& McNamara, 2001). Several recent spatial memory theories (Avraamides \& Kelly, 2008; Sholl, 2001) have proposed that environment-centered, long-term spatial memories are retrieved in an egocentric form conducive to bodybased actions; this is conceptually similar to the spatial framework model (Franklin \& Tversky, 1990). The present research project evaluates some of the evidence supporting this proposal.

Perspective-taking performance is commonly used as an index of the organizational properties of spatial memory. In a typical experiment, participants learn a layout of objects like that depicted in Figure 1. Participants later imagine standing in a specific location (in this case, the center of the layout, indicated by the "+"), imagine facing one (orienting) object, and then point to another (target) object from that perspective. Perspective-taking results have been interpreted as evidence that environmentcentered, long-term spatial memories are retrieved egocentrically (Sholl, 2001). Of particular relevance is the finding that pointing is faster and more accurate when the target object is in the anterior as opposed to the posterior half of space from the imagined perspective. For example, a participant who imagines facing the bear in Figure 1 would be faster and more accurate when pointing to the wolf (in the anterior half of space) than when pointing to the frog (in the posterior half). As noted by Sholl, this asymmetric retrieval of spatial memory, referred to herein as front facilitation, is consistent with other asymmetric properties of the human body: The visual system detects information in front of the head, and the arms and legs are biomechanically suited to act on locations in front of the body. Thus, front facilitation has been considered to be evidence for egocentric retrieval of spatial memory. Front facilitation occurs when the participant is physically located within the to-be-imagined environment, referred to herein as situated retrieval (Sholl, 1987), as well as when the participant is not physically located within the to-be-imagined environment, referred to herein as remote retrieval (Hintzman, O'Dell, \& Arndt, 1981; Shelton \& McNamara, 1997; Sholl, 1987, 1999; Werner \& Schmidt, 1999).

However, aspects of the perspective-taking task itself could be responsible for front facilitation. Rather than resulting from egocentric retrieval, front facilitation might be due to spatial priming from the orienting object. Specifically, object recognition is faster when preceded by presentation of nearby objects than when preceded by that of distant objects (McNamara, 1986). Because perspective-taking experiments typically present the orienting object in front of the body from the imagined perspective ("Imagine facing the bear"), the orienting object might prime other objects in front of the imagined

J.W. Kelly, jonathan.kelly@vanderbilt.edu 


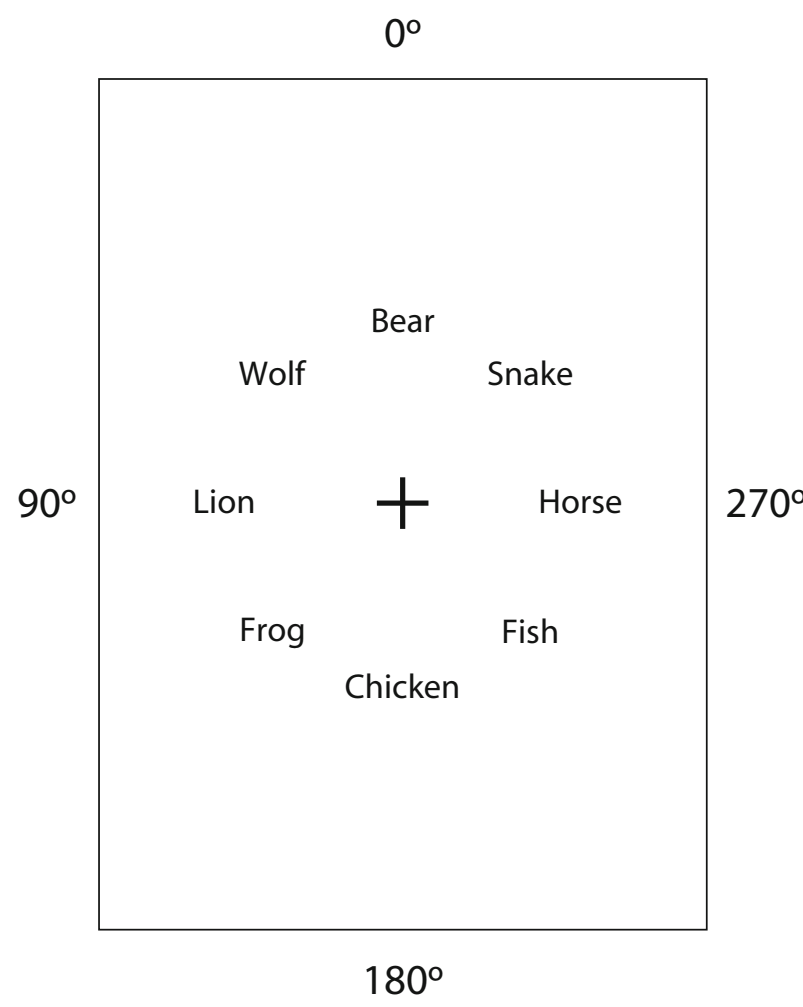

Figure 1. Plan view of the objects and surrounding room used in Experiments 1 and 2. The learning location is represented by the "+."

perspective (objects near the orienting object) more than it does objects behind the imagined perspective (objects far from the orienting object). Furthermore, stimulusresponse compatibility, whereby spatial properties of stimuli can prime responses with similar spatial characteristics (Proctor \& Vu, 2006), might also contribute to this spatial priming effect. In the present experiments, we do not distinguish between priming that is due to shared spatial properties between orienting and target objects and that which is due to shared spatial properties between orienting objects and pointing responses.

Experiments by Hintzman et al. (1981) lend credence to the idea that front facilitation is at least partially due to spatial priming. In one experiment, participants imagined different perspectives by imagining the orienting object to their side, in comparison with the traditional method of imagining facing the orienting object. If front facilitation is due entirely to spatial priming, then presenting the orienting object to the left, for example, should result in facilitated pointing to the left and in equivalent retrieval of objects in front and back. In fact, responses were 33\% faster ${ }^{1}$ when the target object was on the same side than when it was on the opposite side as the orienting object, indicating spatial priming by the orienting object. Furthermore, responses were $10 \%$ faster for target objects in front of, rather than behind, the imagined perspective, indicating front facilitation, even when controlling for spatial priming. However, the statistics necessary for evaluating front facilitation were not presented, and the authors' conclu- sions were based only on the larger spatial priming effect, downplaying the comparatively smaller front facilitation.

In the present experiments, employing a paradigm similar to that of Hinztman et al. (1981), blindfolded participants imagined perspectives within a learned layout. Orienting objects were presented to the sides of the imagined perspectives ("Imagine the lion is to your left. Point to the wolf"), allowing for separate evaluation of front facilitation and spatial priming. Facilitated pointing to target objects in front of the imagined perspective, in comparison with target objects behind the imagined perspective, would indicate front facilitation, a key piece of evidence in support of egocentric spatial memory retrieval. Facilitated pointing to target objects on the same side as the orienting object, rather than to those on the opposite side, would indicate spatial priming. These effects are not mutually exclusive.

In Experiment 1, we sought to determine whether front facilitation - which has been interpreted as being evidence for egocentric retrieval of long-term spatial memorieswould occur during situated retrieval. Objects within the surrounding environment are particularly relevant to egocentric, body-based actions. It is reasonable to think, then, that such egocentric actions - such as reaching for a cup of coffee or walking through a doorway-depend on egocentric spatial representations, and that front facilitation should, therefore, be most likely to occur during situated retrieval. In contrast, objects in remote environments are less relevant to body-based actions, and egocentric representation of their locations is unnecessary. In Experiment 2, we explored whether front facilitation would occur under such remote retrieval conditions.

\section{EXPERIMENT 1}

In Experiment 1, participants studied a layout from a fixed position and orientation (in Figure 1, standing at the "+," facing the bear) but were allowed to turn their heads during learning. After learning, they donned a blindfold, rotated $90^{\circ}$ to their left or right (for this example, let us assume that they turned to the right and were therefore facing the horse), and made pointing judgments from imagined perspectives within the surrounding environment. The ease with which different perspectives within the surrounding environment could be imagined would depend on their alignment with reference directions in long-term memory and body orientation during retrieval (Kelly, Avraamides, \& Loomis, 2007; Kelly \& McNamara, 2008a; Mou, McNamara, Valiquette, \& Rump, 2004). Because either of those two factors could have influenced front facilitation, participants were asked to imagine (1) the learning perspective (aligned with the learning view but misaligned with the body: "Imagine the lion is to your left"), (2) the body-aligned perspective (aligned with the body but misaligned with the learning view: "Imagine the bear is to your left"), and (3) the misaligned perspective (misaligned with the learning view and the body: "Imagine the chicken is to your left"). The body-aligned perspective seemed prima facie most likely to result in front facilitation, since body-based actions are necessarily executed from a body-aligned perspective. 
A control experiment verified that any front facilitation found in these experiments was not due to the pointing device itself. Participants pointed in directions indicated by arrows presented on a monitor. For consistency with Experiments 1 and 2, only front-left, front-right, backleft, and back-right responses were analyzed. Forward and backward pointing were equally fast and accurate, as indicated by pointing latency [front, $M=804 \mathrm{msec}, S E=46$; back, $M=805 \mathrm{msec}, S E=35 ; t(10)=0.021, p=.983]$ and absolute error [front, $M=7.62^{\circ}, S E=1.25$; back, $\left.M=7.08^{\circ}, S E=0.92 ; t(10)=0.752, p=.469\right]$.

\section{Method}

Participants. Sixteen adults ( 8 female) from the Nashville community participated for monetary compensation. Mean age was 23.6 years.

Stimuli and Design. The layout comprised eight objects spaced every $45^{\circ}$ around a $3-\mathrm{m}$-diameter circle centered within a $5 \times 7 \mathrm{~m}$ room (Figure 1). Participants stood in the center of the layout. They faced the bear $\left(0^{\circ}\right)$ during learning, and then, prior to testing, turned $90^{\circ}$ to their left or right to face the lion $\left(90^{\circ}\right)$ or the horse $\left(270^{\circ}\right)$, respectively. Test trials comprised the names of two objects: an orienting object and a target object. Participants imagined that the orienting object was to their side (e.g., "Imagine the horse is to your right"), and then located the target object from that perspective. Target objects of interest were those to the front-left, front-right, backleft, and back-right of the imagined perspective. ${ }^{2}$

The primary independent variables were imagined perspective, cue proximity, and egocentric direction. Imagined perspective could be the learning perspective $\left(0^{\circ}\right)$, the body-aligned perspective $\left(90^{\circ}\right.$ when facing $90^{\circ}$, or $270^{\circ}$ when facing $270^{\circ}$ ), or the misaligned perspective $\left(90^{\circ}\right.$ when facing $270^{\circ}$, or $270^{\circ}$ when facing $\left.90^{\circ}\right)$. Cue proximity could be near (e.g., when the orienting object was on the left and the target object was front-left or back-left from the imagined perspective) or far (e.g., when the orienting object was on the left and the target object was front-right or back-right). Egocentric direction could be front (when the target object was front-left or frontright from the imagined perspective) or back (when the target object was back-left or back-right).

Trials were split into four blocks of 48 trials. Each block contained factorial combinations of imagined perspective, cue proximity, and egocentric direction (which were repeated three times), plus 12 filler trials (to prevent stereotyped responses) with target objects directly in front, in back, and to the sides. Each block was pseudorandomized, so that the same imagined perspective never occurred twice in a row. Two blocks presented the orienting object on the left, and two presented the orienting object on the right (order was counterbalanced). Dependent measures were pointing latency and absolute angular error. Data were recorded on a laptop using Vizard software (WorldViz, Santa Barbara, CA).

Procedure. After providing informed consent, participants were blindfolded and led into the experiment room. The blindfold was removed once the participants were positioned in the center of the layout, facing $0^{\circ}$. Participants were instructed not to move their feet during learning, and to rotate their heads to view the objects. After studying for $60 \mathrm{sec}$, participants closed their eyes and pointed to all objects in a random order. This study-test sequence continued until participants accurately pointed to all objects twice (judged visually by the experimenter).

After learning, participants sat in a swivel chair in the center of the layout and donned a blindfold and headphones, which delivered auditory trial instructions. A wireless joystick (Logitech Freedom 2.4) affixed to a small board was placed on their laps. Prior to testing, participants turned $90^{\circ}$ to their left or right. Order of physical facing direction was counterbalanced across blocks. Each trial described the to-be-imagined perspective by presenting the orienting object to the side. Responses were recorded when the joystick was deflected $30^{\circ}$ from vertical. After each trial block, participants returned to face $0^{\circ}$ and reviewed the layout.

\section{Results and Discussion}

Pointing latency was more responsive to the independent variables than was angular error, but the two variables were positively correlated $(r=.50)$ and showed no evidence of a speed-accuracy trade-off. For brevity, we focus on latency.

Pointing latency (Figure 2) was analyzed in a repeated measures ANOVA with terms for imagined perspective (learning, body-aligned, or misaligned), cue proximity (target object near or far from the orienting object), and egocentric direction (front or back). A main effect of imagined perspective $\left[F(2,30)=21.34, p<.001, \eta_{\mathrm{p}}^{2}=\right.$ .59] indicated faster pointing from the body-aligned perspective $[M=2.70 \mathrm{sec}, S E=.38]$ than from the learning perspective $(M=3.79 \mathrm{sec}, S E=0.53)[F(1,15)=14.30$, $\left.p=.002, \eta_{\mathrm{p}}^{2}=.49\right]$, which was faster than pointing from the misaligned perspective $(M=4.96 \mathrm{sec}, S E=0.73)$ $\left[F(1,15)=27.22, p<.001, \eta_{\mathrm{p}}^{2}=.65\right]$. A main effect of cue proximity $\left[F(1,15)=15.09, p=.001, \eta_{\mathrm{p}}^{2}=.50\right]$ indicated that spatial priming occurred; that is, there was faster pointing to target objects on the same side as the orienting object $(M=3.35 \mathrm{sec}, S E=0.43)$ than to those on the opposite side $(M=4.28 \mathrm{sec}, S E=0.63)$. A main effect of egocentric direction $[F(1,15)=11.90, p=.004$, $\left.\eta_{\mathrm{p}}^{2}=.44\right]$ indicated front facilitation; that is, there was

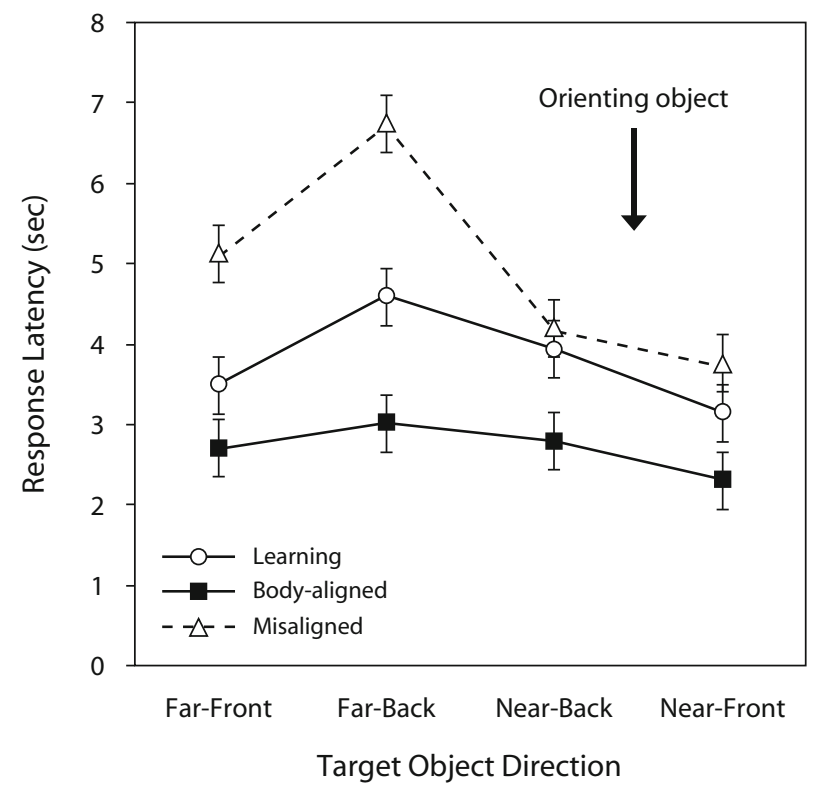

Figure 2. Response latencies in Experiment 1, as a function of target object direction, plotted separately for the three imagined perspectives. The target object could be on the same side of the imagined perspective as the orienting object (near; e.g., when the orienting and target objects were both to the left) or on the opposite side (far; e.g., when the orienting object was on the left and the target object was on the right). Additionally, the target object could be in either the anterior half of space from the imagined perspective (front) or in the posterior half (far). The arrow indicates the orienting object's location. Error bars are standard errors estimated from the ANOVA. 
faster pointing to target objects in front $(M=3.42 \mathrm{sec}$, $S E=0.44)$ than to those behind $(M=4.21 \mathrm{sec}, S E=$ $0.62)$. Imagined perspective interacted with cue proximity $\left[F(2,30)=7.51, p=.002, \eta_{\mathrm{p}}^{2}=.33\right]$; spatial priming was greater for imagining the misaligned $(1.96 \mathrm{sec})$ perspective than for imagining the learning $(0.50 \mathrm{sec})$ $\left[F(1,15)=8.16, p=.012, \eta_{\mathrm{p}}^{2}=.35\right]$ or body-aligned $(0.31 \mathrm{sec})\left[F(1,15)=7.89, p=.013, \eta_{\mathrm{p}}^{2}=.35\right]$ perspective. No other effects were significant.

Front facilitation occurred from all three imagined perspectives, even when controlling for spatial priming effects. Consistent with asymmetrical sensory and biomechanical properties of the human body, front facilitation supports the notion that spatial memories are retrieved egocentrically during situated retrieval; this notion is an important component of recent spatial memory theories (Avraamides \& Kelly, 2008; Sholl, 2001).

Spatial priming also influenced spatial memory retrieval; pointing was faster for target objects near the orienting object than for those that were far from it. This was particularly true when participants imagined the misaligned perspective, for which overall latencies were greatest. Even when spatial priming is expressed as a percentage (calculated as percent latency reduction when pointing to near, compared with far, objects), priming was larger for the misaligned (33.0\%) perspective than for the learning (12.4\%) or body-aligned (10.9\%) perspective.

Similar to findings from previous work (Kelly et al., 2007; Kelly \& McNamara, 2008a; Mou et al., 2004), pointing was faster for imagining the learning perspective or the body-aligned perspective than for imagining the misaligned perspective. Facilitated pointing from the learning perspective is thought to result from organizational properties of long-term spatial memory. According to Shelton and McNamara (2001), reference directions are selected on the basis of cues in the learning environment, and those reference directions serve to organize the longterm representation. Participants faced a single orientation during learning (although they were free to rotate their heads to inspect the layout), and this facing direction coincided with the long axis of the room. One or both of these factors could have contributed to the facilitated responses when participants imagined the learning perspective. Facilitated pointing from the body-aligned perspective in comparison with pointing from the misaligned perspective is thought to be due to an egocentric sensorimotor representation of the environment. The sensorimotor representation is updated during self-motion and is thought to underlie actions such as obstacle avoidance. Additionally, the sensorimotor representation interferes with the ability to imagine perspectives misaligned with the body (May, 2004) and must be suppressed in order for one to imagine the environment in new egocentric coordinates.

The front facilitation found in Experiment 1 supports the claim that spatial memories of the surrounding environment are retrieved egocentrically (Sholl, 2001). However, spatial memories of remote environments may be retrieved quite differently from those of the surrounding environment, since memories of remote environments are less relevant for body-based actions. In Experiment 2, we tested for front facilitation during remote retrieval of spatial memories.

\section{EXPERIMENT 2}

Whereas Experiment 1 indicated that spatial memories of the surrounding environment are retrieved egocentrically, the purpose of Experiment 2 was to explore whether spatial memories of remote environments are also retrieved egocentrically. After learning, participants were led to a remote location for retrieval.

\section{Method}

Participants. Sixteen adults ( 8 female) from the Nashville community participated for monetary compensation. Mean age was 24.2 years.

Stimuli, Design, and Procedure. Learning was identical to that in Experiment 1. After learning, participants were led to another room on a different floor of the building, where they were seated and donned the blindfold and headphones. The same imagined perspectives from Experiment 1 were used in Experiment 2, but because participants no longer physically occupied the learning environment (and so body alignment was irrelevant), the imagined perspectives were then referred to in environment-centered terms: $0^{\circ}$ (the learning perspective), $90^{\circ}$, and $270^{\circ}$. As in Experiment 1, testing occurred over four blocks of 48 trials. Each block comprised factorial combinations of imagined perspective $\left(0^{\circ}, 90^{\circ}\right.$, or $\left.270^{\circ}\right)$, cue proximity (near or far), and egocentric direction (front or back)-repeated three times - plus 12 filler trials.

\section{Results and Discussion}

Latency was more responsive to the independent variables than was angular error, and there was no evidence of a speed-accuracy trade-off: latency and error were positively correlated $(r=.23)$. For brevity, we focus on latency.

Pointing latency (Figure 3) was analyzed in a repeated measures ANOVA with terms for imagined perspective $\left(0^{\circ}, 90^{\circ}\right.$, or $\left.270^{\circ}\right)$, cue proximity (near or far), and egocentric direction (front or back). A main effect of imagined perspective $\left[F(2,30)=16.80, p<.001, \eta_{\mathrm{p}}^{2}=.53\right]$ indicated faster responses for imagining the $0^{\circ}$ perspective $(M=2.87 \mathrm{sec}, S E=0.44)$ than for imagining the $90^{\circ}$ $[M=4.55 \mathrm{sec}, S E=0.61 ; F(1,15)=20.72, p<.001$, $\left.\eta_{\mathrm{p}}^{2}=.58\right]$ or $270^{\circ}[M=4.19 \mathrm{sec}, S E=0.52 ; F(1,15)=$ $\left.15.37, p=.001, \eta_{\mathrm{p}}^{2}=.51\right]$ perspective. A main effect of cue proximity $\left[F(1,15)=6.52, p=.022, \eta_{\mathrm{p}}^{2}=.30\right]$ indicated faster responses to target objects on the same side as the orienting object $(M=3.55 \mathrm{sec}, S E=0.45)$ than to those on the opposite side $(M=4.19 \mathrm{sec}, S E=0.57)$. A main effect of egocentric direction $[F(1,15)=19.59$, $\left.p<.001, \eta_{\mathrm{p}}^{2}=.57\right]$ indicated faster responses to target objects in front of the imagined perspective $(M=3.45 \mathrm{sec}$, $S E=0.43)$ than to those behind $(M=4.29 \mathrm{sec}, S E=$ $0.58)$. Imagined perspective interacted with cue proximity $\left[F(2,30)=4.99, p=.013, \eta_{\mathrm{p}}^{2}=.25\right]$, where the benefit of pointing to objects near the orienting object was larger for imagining the $90^{\circ}(0.89 \mathrm{sec})$ and $270^{\circ}(0.94 \mathrm{sec})$ perspectives than for imagining the $0^{\circ}(0.09 \mathrm{sec})$ perspective. No other effects were significant.

Responses from all three imagined perspectives were faster for pointing to objects in front of the imagined perspective than for pointing to those behind it. This front 


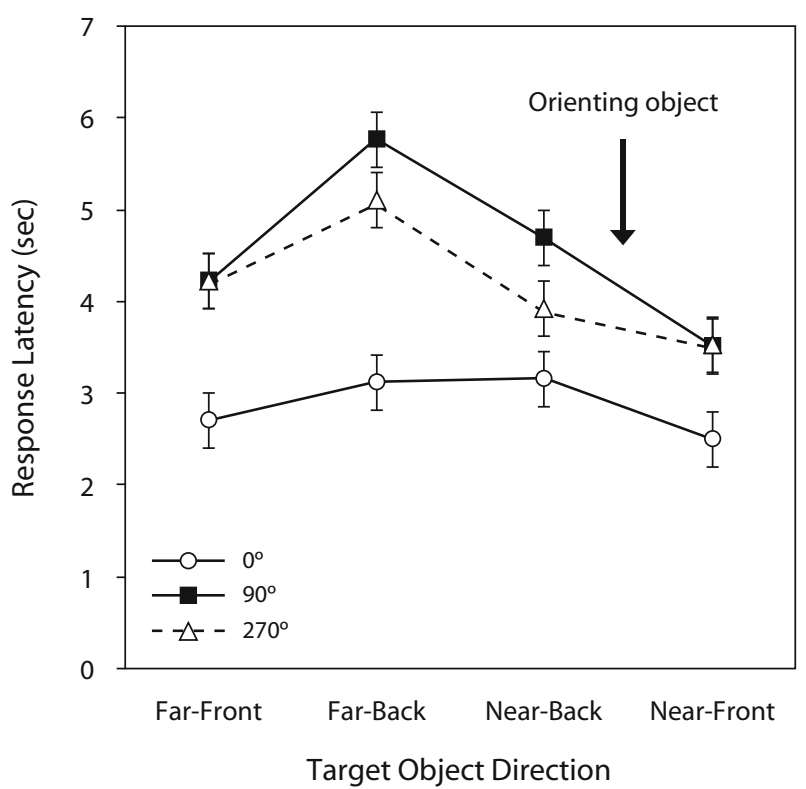

Figure 3. Response latencies in Experiment 2, as a function of the target object direction, plotted separately for the three imagined perspectives. The target object could be on the same side of the imagined perspective as the orienting object (near; e.g., when the orienting and target objects were both to the left) or on the opposite side (far; e.g., when the orienting object was on the left and the target object was on the right). Additionally, the target object could be in either the anterior half of space from the imagined perspective (front) or in the posterior half (far). The arrow indicates the orienting object's location. Error bars are standard errors estimated from the ANOVA.

facilitation supports theories that claim that spatial memories of remote environments are retrieved egocentrically (Avraamides \& Kelly, 2008; Sholl, 2001). This is somewhat surprising because remembered locations within a remote environment cannot be egocentrically acted upon without one's first having traveled to that environment. Front facilitation during remote retrieval might indicate that retrieval processes during situated and remote retrieval share common neural structures (Sholl, 2001), a possibility pursued in more detail in the General Discussion.

Spatial priming occurred when participants imagined the $90^{\circ}$ and $270^{\circ}$ perspectives, but it is unclear why it was reduced when they imagined the learning perspective. This finding is inconsistent with that of Experiment 1, where spatial priming occurred for the learning perspective. Similar to what we found in Experiment 1, the relative ease with which participants imagined the $0^{\circ}$ perspective indicates that their spatial memories were organized around a reference direction coincident with the learning direction and primary-room axis.

The delay between learning and testing was slightly longer under the remote testing conditions of Experiment 2 than under the situated testing conditions of Experiment 1, due to the additional time (1-2 $\mathrm{min}$ ) required to walk to the remote testing location. It is unclear what effect this delay might have had on performance, but overall latencies were similar in Experiments $1(3.81 \mathrm{sec})$ and $2(3.87 \mathrm{sec})$.

\section{GENERAL DISCUSSION}

Several recent spatial memory theories posit that longterm spatial memories are retrieved egocentrically during situated and remote retrieval (Avraamides \& Kelly, 2008; Sholl, 2001). One source of evidence for egocentric retrieval is that pointing to objects from imagined perspectives within a remembered environment is facilitated for objects in front of, rather than for those behind, the imagined perspective (Shelton \& McNamara, 1997; Sholl, 1987, 1999; Werner \& Schmidt, 1999). However, experiments by Hintzman et al. (1981) suggest that front facilitation might be due to spatial priming. By presenting orienting objects to the sides of imagined perspectives, thereby unconfounding front facilitation and spatial priming, Hintzman et al. found evidence for spatial priming and concluded that spatial priming fully accounted for the response profile. However, this conclusion does not completely correspond with their results, which showed a $10 \%$ latency reduction for pointing to the front.

The present experiments directly assessed front facilitation while controlling for spatial priming. Front facilitation occurred during situated (Experiment 1) and remote (Experiment 2) testing, supporting the proposal that spatial memories are retrieved egocentrically. According to Sholl (2001), spatial memories of surrounding or remote environments are retrieved within an egocentric self-reference system. During situated retrieval, the self-reference system operates as a sensorimotor self-reference system, which has a preferred orientation consistent with one's body orientation. During remote retrieval, the self-reference system operates as a representational self-reference system. Sholl considered long-term spatial memories to be orientation independent, and so any perspective should be equally accessible through the representational self-reference system. However, results from the present experiments and others (Mou \& McNamara, 2002; Shelton \& McNamara, 2001) indicate that spatial memories are orientation dependent.

Egocentric spatial memory retrieval provides an isomorphic mapping between representation and response, allowing for facilitated execution of memory-guided actions. The front facilitation found here is consistent with the body's sensory and biomechanical asymmetries. This makes sense during situated retrieval, when egocentric actions can be directed toward surrounding objects. But why did front facilitation also occur during remote retrieval, when the retrieved objects could not be acted on egocentrically from the remote environment? According to Sholl (2001), the sensorimotor and representational self-reference systems (active during situated and remote retrieval, respectively) share overlapping neural architecture. As a result, the representational system is imbued with the same egocentric properties as the sensorimotor system. One potential benefit of this shared architecture is that egocentric retrieval of a remote space could be useful for planning future actions within that space. Furthermore, this shared architecture may underlie the findings of May (2007), where imagined perspec- 
tive changes (e.g., imagining a $135^{\circ}$ rotation) resulted in similar performance costs under situated and remote test conditions.

In sum, the present experiments indicate that long-term, orientation-dependent spatial memories are retrieved egocentrically during both situated and remote retrieval. This egocentric retrieval reflects the fundamental role of spatial memories in supporting the body-based actions necessary for navigation.

\section{AUTHOR NOTE}

This work was supported by National Institute of Mental Health Grant 2-R01-MH57868. Correspondence concerning this article should be addressed to J. W. Kelly, Department of Psychology, Vanderbilt University, 111 21st Ave. S., Nashville, TN 37203 (e-mail: jonathan.kelly@ vanderbilt.edu).

\section{REFERENCES}

Avraamides, M. N., \& Kelly, J. W. (2008). Multiple systems of spatial memory and action. Cognitive Processing, 9, 93-106. doi:10.1007/ s10339-007-0188-5

Franklin, N., \& TVersky, B. (1990). Searching imagined environments. Journal of Experimental Psychology: General, 119, 63-76. doi:10.1037/0096-3445.119.1.63

Hintzman, D. L., O’Dell, C. S., \& Arndt, D. R. (1981). Orientation in cognitive maps. Cognitive Psychology, 13, 149-206. doi:10.1016/0010 $-0285(81) 90007-4$

Kelly, J. W., Avraamides, M. N., \& Loomis, J. M. (2007). Sensorimotor alignment effects in the learning environment and in novel environments. Journal of Experimental Psychology: Learning, Memory, \& Cognition, 33, 1092-1107. doi:10.1037/0278-7393.33.6.1092

Kelly, J. W., \& McNamara, T. P. (2008a). Response mode differences in perspective taking: Differences in representation or differences in retrieval? Memory \& Cognition, 36, 863-872. doi:10.3758/ MC.36.4.863

Kelly, J. W., \& McNamara, T. P. (2008b). Spatial memories of virtual environments: How egocentric experience, intrinsic structure, and extrinsic structure interact. Psychonomic Bulletin \& Review, 15, 322327. doi:10.3758/PBR.15.2.322

MAY, M. (2004). Imaginal perspective switches in remembered environments: Transformation versus interference accounts. Cognitive Psychology, 48, 163-206. doi:10.1016/S0010-0285(03)00127-0

MAY, M. (2007). Imaginal repositioning in everyday environments: Effects of testing method and setting. Psychological Research, 71, 277287. doi:10.1007/s00426-006-0083-1

MCNamara, T. P. (1986). Mental representations of spatial relations. Cognitive Psychology, 18, 87-121. doi:10.1016/0010-0285(86)90016-2
Mou, W., \& McNamara, T. P. (2002). Intrinsic frames of reference in spatial memory. Journal of Experimental Psychology: Learning, Memory, \& Cognition, 28, 162-170. doi:10.1037/0278-7393.28.1.162

Mou, W., McNamara, T. P., Valiquette, C. M., \& Rump, B. (2004). Allocentric and egocentric updating of spatial memories. Journal of Experimental Psychology: Learning, Memory, \& Cognition, 30, 142157. doi: $10.1037 / 0278-7393.30 .1 .142$

Proctor, R. W., \& Vu, K.-P. L. (2006). Stimulus-response compatibility principles: Data, theory, and application. Boca Raton, FL: CRC Press.

Rump, B., \& McNamara, T. P. (2007). Updating in models of spatial memory. In. T. Barkowsky, M. Knauff, G. Ligozat, \& D. R. Montello (Eds.), Spatial cognition V: Reasoning, action, interaction (pp. 249269). Berlin: Springer. doi:10.1007/978-3-540-75666-8

Shelton, A. L., \& McNamara, T. P. (1997). Multiple views of spatial memory. Psychonomic Bulletin \& Review, 4, 102-106.

Shelton, A. L., \& McNamara, T. P. (2001). Systems of spatial reference in human memory. Cognitive Psychology, 43, 274-310. doi:10.1006/ cogp. 2001.0758

SHoll, M. J. (1987). Cognitive maps as orienting schemata. Journal of Experimental Psychology: Learning, Memory, \& Cognition, 13, 615628. doi:10.1037/0278-7393.13.4.615

SHOLL, M. J. (1999). Egocentric frames of reference used for the retrieval of survey knowledge learned by map and navigation. Spatial Cognition \& Computation, 1, 475-494. doi:10.1023/A:1010047911951

SHoLl, M. J. (2001). The role of a self-reference system in spatial navigation. In D. R. Montello (Ed.), Spatial information theory: Foundations of geographic information science (pp. 217-232). Berlin: Springer. doi:10.1007/3-540-45424-1_15

Werner, S., \& Schmidt, K. (1999). Environmental reference systems for large-scale spaces. Spatial Cognition \& Computation, 1, 447-473. doi:10.1023/A:1010095831166

\section{NOTES}

1. This value was estimated from Hintzman et al. (1981, Figure 18). For consistency with the present experiments, this calculation only considered front-left, front-right, back-left, and back-right responses. Spatial priming was calculated by comparing latencies for target objects on the same side as the orienting object versus latencies on the side opposite the orienting object. Front facilitation was calculated by comparing latencies for target objects in front versus back.

2. Rump and McNamara (2007) reported facilitated pointing in directions parallel to salient room axes. In the present experiments, this effect was circumvented by using target objects requiring oblique pointing responses. Other target object locations were also included, but served only as filler trials to prevent stereotyped responses.

(Manuscript received July 2, 2008; revision accepted for publication September 8, 2008.) 